\title{
Risk Factors for Liver Cirrhosis-Related Readmissions in the Largest Ethnic Minority in United States
}

\author{
Harish Patel ${ }^{\mathrm{a}}$, Bhavna Balar ${ }^{\mathrm{a}}$, Maheswara Irigela ${ }^{\mathrm{a}}$, Vamshidhar Vootla ${ }^{\mathrm{a}}$, Chaitanya Chandrala ${ }^{\mathrm{a}}$, \\ Hafiz Hashmi ${ }^{a}$, Molham Abdulsamad ${ }^{a}$, Jasbir Makker ${ }^{a}$, b
}

\begin{abstract}
Background: There are very limited data available on 30-day readmissions for ethnic minority patients with cirrhosis. The aim of the study was to identify the risk factors for 30-day readmission in ethnic minority patients admitted for cirrhosis.
\end{abstract}

Methods: We did a retrospective review of 1,373 electronic medical records of patients with cirrhosis admitted from 2009 to 2011. Several parameters including alcohol use history, discharge location and cirrhosis severity scores - model for end-stage liver disease (MELD) score and Child-Pugh-Turcotte (CPT) at first admission were assessed. Statistical analysis was done using Chi-square test and $t$-test for categorical and continuous variables, respectively.

Results: There were 79 patients in the readmission group (63\% male, 54\% Hispanics and 22\% African Americans) and 104 in the no readmission group (62\% male, 58\% Hispanics and 24\% African Americans). History of alcohol use within a month prior to admission $(55 \%$ vs. $33 \%, P=0.002)$, platelet count at discharge $(89,000$ vs. 124,000 , $\mathrm{P}=0.003)$, and discharge with more than seven medications per day (7.3 vs. 5.2, $\mathrm{P}=0.005$ ) were identified as risk factors for readmissions by multivariate analysis.

Conclusion: Platelet count, active alcohol use and more than seven medications at discharge are predictors of readmission. These parameters can guide future interventions to reduce readmission rate and health care costs related to cirrhosis readmissions.

Keywords: Cirrhosis; Readmission; Hispanics; African Americans; Alcohol

\section{Introduction}

According to United States Census Bureau's survey, 58.9 mil-

Manuscript submitted September 19, 2019, accepted January 10, 2020

aDepartment of Medicine, BronxCare Health System, 1650 Selwyn Ave., Bronx, NY 10457, USA

${ }^{\mathrm{b} C}$ Corresponding Author: Jasbir Makker, Department of Medicine, BronxCare Health System, 1650 Selwyn Ave., Bronx, NY 10457, USA.

Email: makkerjs@gmail.com

doi: https://doi.org/10.14740/gr1227 lion Hispanics accounted for $18.1 \%$ of United States total population and were the largest ethnic minority in United States in 2017. Total population of African Americans in United States in 2017 was 47.4 million. Significant health disparities undermine the health system in Unites States. In 2017, about 17.8\% of Hispanics and $10 \%$ of African Americans had no health insurance [1]. On the other hand, the highest prevalence of cirrhosis is found among Hispanics [2]. Chronic liver disease and cirrhosis ranked as seventh leading cause of death among Hispanics in 2016, while for the Whites and African Americans, cirrhosis-related deaths did not find place in top 10 causes [3].

Liver cirrhosis puts a significant burden on the health care system. National Health and Nutrition Examination Survey data reveal higher prevalence of liver cirrhosis than previously predicted [4]. There were estimated 633,323 individuals with liver cirrhosis between 1993 and 2010. With disease progression, patients with liver cirrhosis present with various features of decompensation. The clinical presentation of decompensated liver cirrhosis involves manifestations of end-organ damage including portal hypertension and porto-systemic shunting leading to hepatic encephalopathy, renal insufficiency, variceal bleeding and uncontrolled ascites [5]. Management of a patient with decompensated liver cirrhosis in an ambulatory setting is a challenge due to complexity of the disease and often requires hospitalization.

Hospitalization of patients with liver cirrhosis incurs a high health care expenditure [6]. A single hospitalization cost is estimated to be $\$ 15,000$ [6], and it carries a $37 \%$ risk of readmission, which further intensifies the cost to $\$ 20,000$ per hospitalized visit [7]. Another study estimated the total cost of all admissions related to decompensated cirrhosis to be 1.8 billion, and an additional 0.5 billion due to readmissions [8]. The Patient Protection and Affordable Care Act has added a new horizon to the value-based health care model. Hospitals are held accountable and often penalized for the readmissions within 30 days of discharge [9]. Center for Medicare and Medicaid Services (CMS) has implemented hospital readmission reduction program (HRRP) that reduces payments for 30-day readmissions related to six conditions or procedures [10]. At this point, readmissions related to liver cirrhosis are not a component of the HRRP. However, readmissions related to any condition are considered a marker of poor health care delivery. Hence, it is imperative for the hospitals to first identify patients with cirrhosis at risk for readmission, and then structure a program utilizing appropriate resources to prevent such readmissions. 
Efforts have been made to identify the risk factors for readmissions related to other diseases like congestive heart failure (CHF) [11]. Various evidence-based interventions targeting those at risk for CHF-related readmissions have been implemented to attain success in reducing the burden of readmission [12]. Akin to CHF, there has been an effort to identify patients with liver cirrhosis-related readmissions [13]. Studies so far in patients with cirrhosis have identified variable risk factors for cirrhosis patients' readmissions. Higher model for end-stage liver disease (MELD) score has been associated with higher rate of readmissions and these readmissions have been linked to higher mortality rates [14].

Our institution serves the underserved minority population in South Bronx with majority of them being Hispanics and African Americans. In our study, we intend to identify the risk factors for 30-day readmissions related to liver cirrhosis in this minority population. This will assist in implementation of adequate measures to prevent the readmissions in this underserved population.

\section{Materials and Methods}

Our study was a retrospective review. The study was performed as per the Declaration of Helsinki and protocol was approved by Institution Review Board (IRB) of BronxCare Health System. The study period was from January 2009 to December of 2011.

\section{Study population}

All hospitalized patients with ICD9 or ICD10 code inclusive of liver cirrhosis or related complications, namely hepatic encephalopathy, variceal bleeding, worsening ascites, spontaneous bacterial peritonitis, hepato-renal syndrome and hepatocellular carcinoma were extracted. The first admission during the study period was considered as the index admission and further readmissions if any were noted. The admission records were reviewed to determine the reason for readmission.

\section{Study groups}

The study population of these patients with liver cirrhosis was divided into two groups based on their readmission within 30 days of discharge. The study groups were named as: 1) Patients with readmission and 2) Patients with no readmission.

\section{Demographic information, co-morbid conditions and laboratory parameters}

The demographic information like age, gender and ethnicity were reviewed from the electronic medical record (EMR). Chart review was also performed to document the co-morbid conditions in these patients. The laboratory parameters at the time of index admission were used for study analysis.

\section{Liver cirrhosis scoring indices and alcohol use}

The MELD score, MELD-sodium (MELD-Na) score and Child-Pugh-Turcotte (CPT) were calculated based on assessment at the time of the index admission. The physician documentation in the EMR was reviewed to obtain the history of alcohol use.

\section{Discharge disposition and number of medications at the time of discharge}

The discharge orders were reviewed for the discharge disposition location, which included three main groups, namely discharge to nursing home, discharge to home with services and discharge to home with no services. The discharge instruction documentation and prescription writer in EMR were reviewed for the number of medications prescribed at the time of discharge. The patients who had death during the index hospital visit or had signed out against the medical advice were not included for the further analysis.

\section{Ambulatory clinic follow-up}

The clinic visits within the hospital and primary care network were reviewed to account for the post-discharge follow-ups. Based on number of days from the time of hospital discharge to clinic follow-up, patients were divided into three groups: 7 days clinic follow-up, 8 - 14 days clinic follow-up and 15 - 30 days clinic follow-up.

\section{Statistical analysis}

The co-morbid conditions, demographic data, laboratory parameter, discharge disposition location and post-discharge follow-up were stratified across the two groups: patients with readmission and patients with no readmission. Categorical variables were reported with frequency and percentage. Continuous variables were reported with mean and standard deviation. P-value less than 0.05 was considered statistically significant.

\section{Results}

A total of 1,373 patient visits were identified and reviewed during the study period from which 960 patient visits had the liver cirrhosis as the primary diagnosis for hospitalization. There were 568 patient visits that had death or discharge against the medical advice as discharge disposition and hence they were excluded from the study (Fig. 1). The study population included remaining 392 patient visits which comprised of 183 unique patients. There were 79 patients in "readmission group" and 104 in "no readmission group".

The baseline characteristics of both the groups were comparable except for hepatitis B status and the platelet count (Ta- 


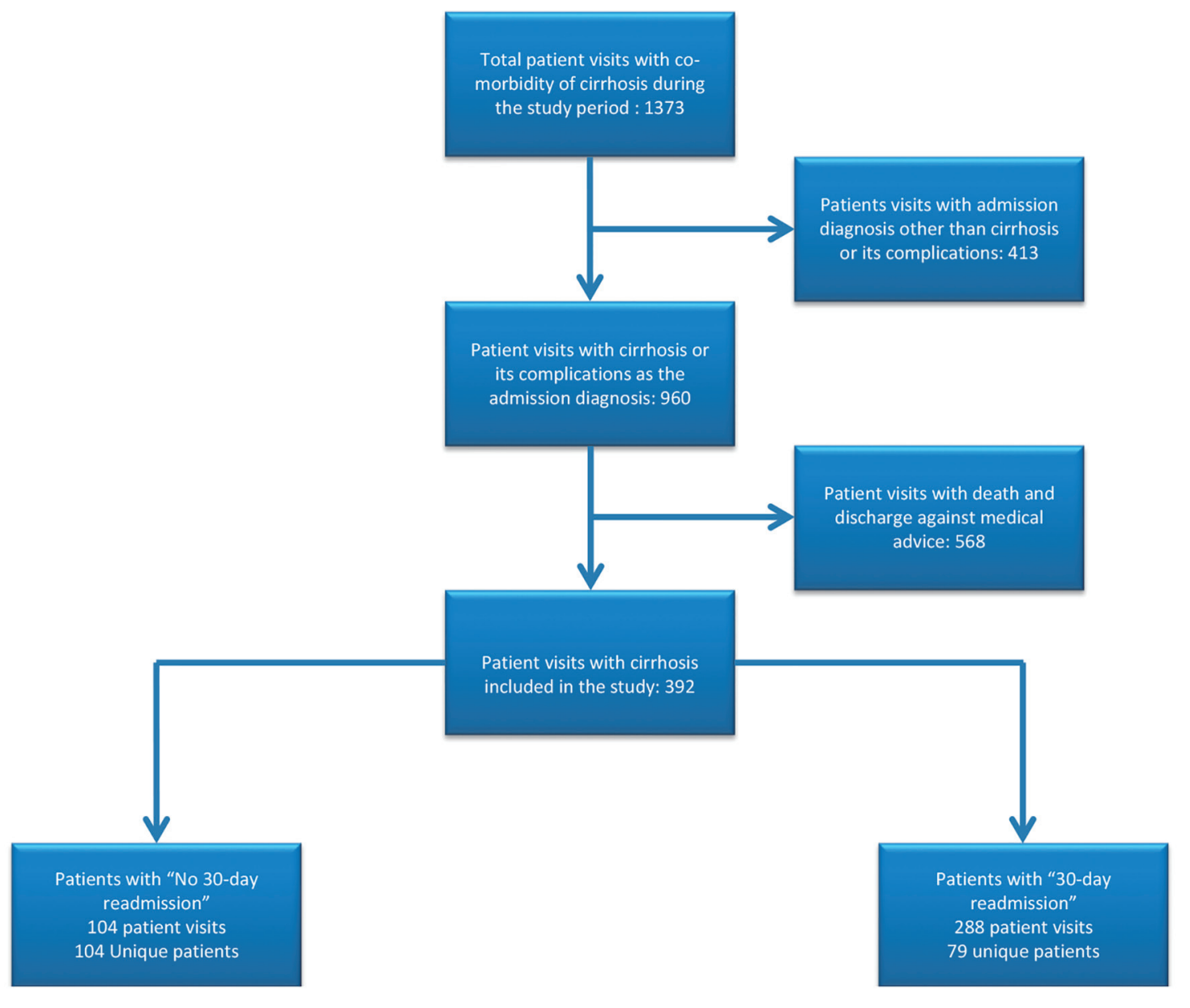

Figure 1. Flowchart of patients included in the study.

ble 1). There was no significant difference in the mean age of the patients between the two groups of no readmission (58.3 years) versus those who had readmission (59.8 years). Majority of them were male in both the groups. There was no significant difference in race distribution between the two groups. Hispanics were predominant in both the groups, followed by African Americans. Patients with chronic hepatitis B were more common in readmission group $(n=5,6.33 \%)$ as compared to the no readmission group $(n=1,0.9 \%)$ and this difference was statistically significant $(\mathrm{P}=0.04)$. There was no significant difference in the other cardiac, pulmonary and oncological co-morbidities. The HIV-positive patients were distributed equally in both the groups. The liver serology including alanine amino transferase (ALT), total bilirubin and prothrombin time were comparable among both the groups. The platelet count of patients in the readmission group at discharge was significantly lower than those with no readmission (89,000 vs. $124,000, \mathrm{P}=0.006)$.

The comparison of MELD score for the readmission and no readmission group revealed no statistical significance (Table 2). MELD score of patients with no readmission was 15 $(+8)$ as compared to $16(+6)$ of those with readmission. However, CPT class did impact the readmission rates. Patients with the CPT class A were less likely to be readmitted than patients with the CPT class B and C. History of active alcohol use was noted as one of the risk factors of readmission. There was a total of $44(55 \%)$ patients with alcohol use within 1 month in readmission group as compared to $34(32.6 \%)$ patients in the no readmission group $(\mathrm{P}=0.002)$.

There were no significant differences when reasons for admission were compared between the two groups (Table 3). Ascites was the predominant indication for the admission in both groups contributing to $45.6 \%$ in the readmission group versus $42.3 \%$ in the no readmission group. Hepatic encephalopathy was the second common cause for the admissions. Those who were discharged to skilled home facility were more likely to have 30 -day readmissions. Only a few patients were discharged to their home with no services $(n=4)$ whereas discharge disposition to home with home care services was equally distributed in both the groups (Table 3). Number of medications prescribed at the time of discharge from the hospital was a significant risk factor for readmission. Patients with seven or more medications per day at the time of discharge were at higher risk for readmissions $(\mathrm{P}=0.005)$.

We further analyzed the group of patients with readmissions. We compared the reason for hospitalization at their 
Table 1. Baseline Demographic, Co-Morbid Conditions and Laboratory Parameters Stratified Between the Two Groups

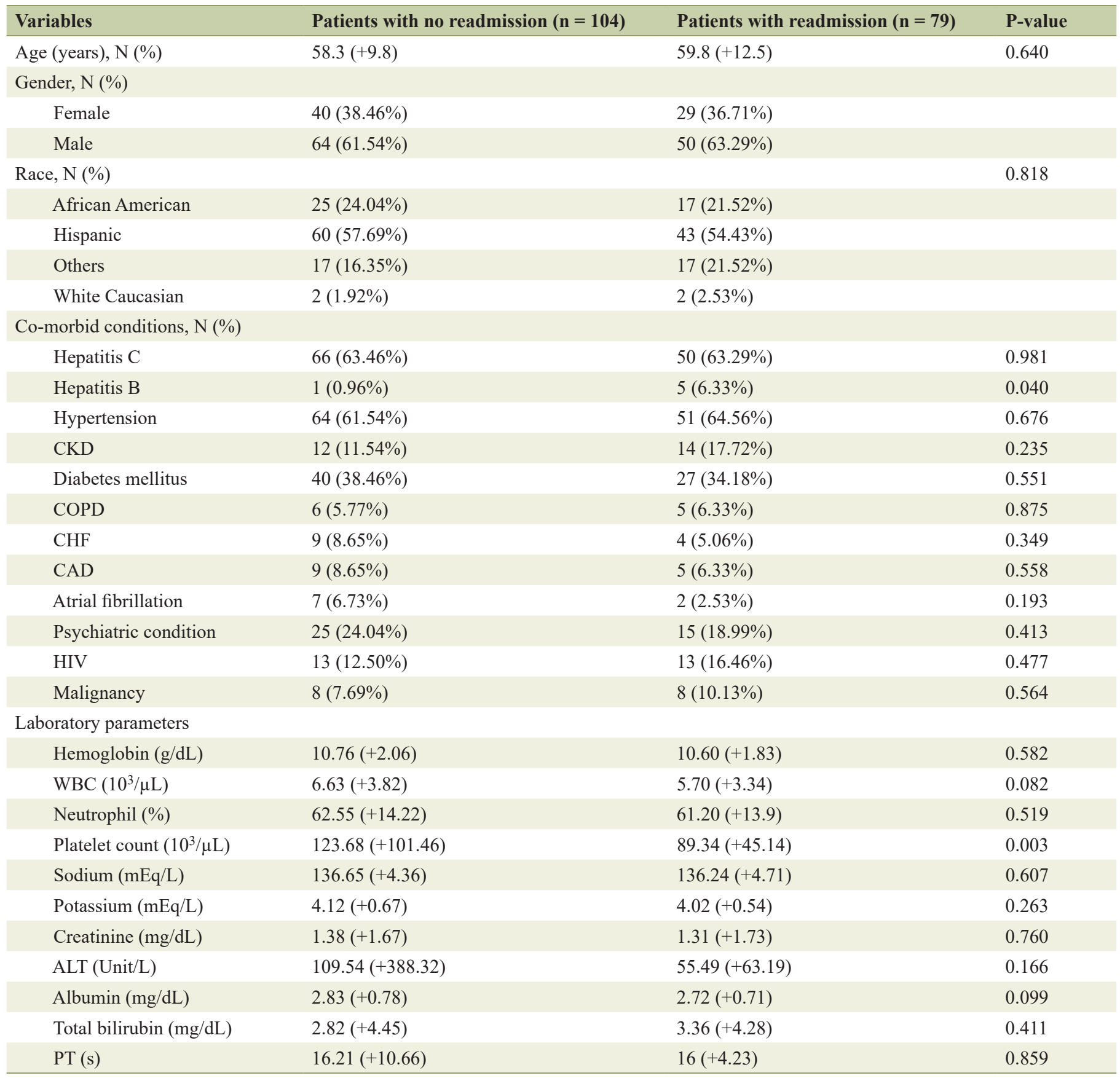

CKD: chronic kidney disease; COPD: chronic obstructive pulmonary disease; CHF: congestive heart failure; CAD: coronary artery disease; WBC: white blood cell count; ALT: alanine aminotransferase; PT: prothrombin time.

index hospitalization to the reason for hospitalization during their subsequent visit (Table 4). Ascites and hepatic encephalopathy were the two most common reasons for hospitalization leading to readmissions. There was no statistical difference when the reason for hospitalization was compared among index and subsequent hospitalization in the readmission group.

The post-hospital discharge ambulatory follow-up did not have significant impact to prevent the 30-day readmission
(Table 5). The post-discharge clinic follow-ups within 7 days, 8 - 14 days and 15 - 30 days were evenly distributed in both the groups. From the patients who had follow-up in the ambulatory clinic, majority of them were evaluated by primary care physicians with $23(22.1 \%)$ patients in the no readmission group as opposed to $17(21.5 \%)$ patients in the readmission group. There was no significant difference in the 90-day mortality between both the groups. 
Table 2. Liver Cirrhosis Scoring and Alcohol Use in Relation to Readmission

\begin{tabular}{|c|c|c|c|}
\hline & $\begin{array}{l}\text { Patients with no readmission } \\
(n=104)\end{array}$ & $\begin{array}{l}\text { Patients with readmission } \\
(\mathrm{n}=79)\end{array}$ & P-value \\
\hline MELD score & $15 \pm 8$ & $16 \pm 6$ & 0.250 \\
\hline Child-Pugh class (CPT) & & & 0.013 \\
\hline CPT class A, N (\%) & $6(13.33 \%)$ & $0(0.00 \%)$ & \\
\hline Alcohol use within 1 month of admission, $\mathrm{N}(\%)$ & $34(32.69 \%)$ & $44(55 \%)$ & 0.002 \\
\hline Alcohol use between 1 and 6 months prior to admission, $\mathrm{N}(\%)$ & $32(30.7 \%)$ & $33(41 \%)$ & 0.123 \\
\hline
\end{tabular}

MELD: model for end-stage liver disease; Na: sodium.

Table 3. Reason for Admission, Discharge Disposition and Number of Medications at the Time of Discharge

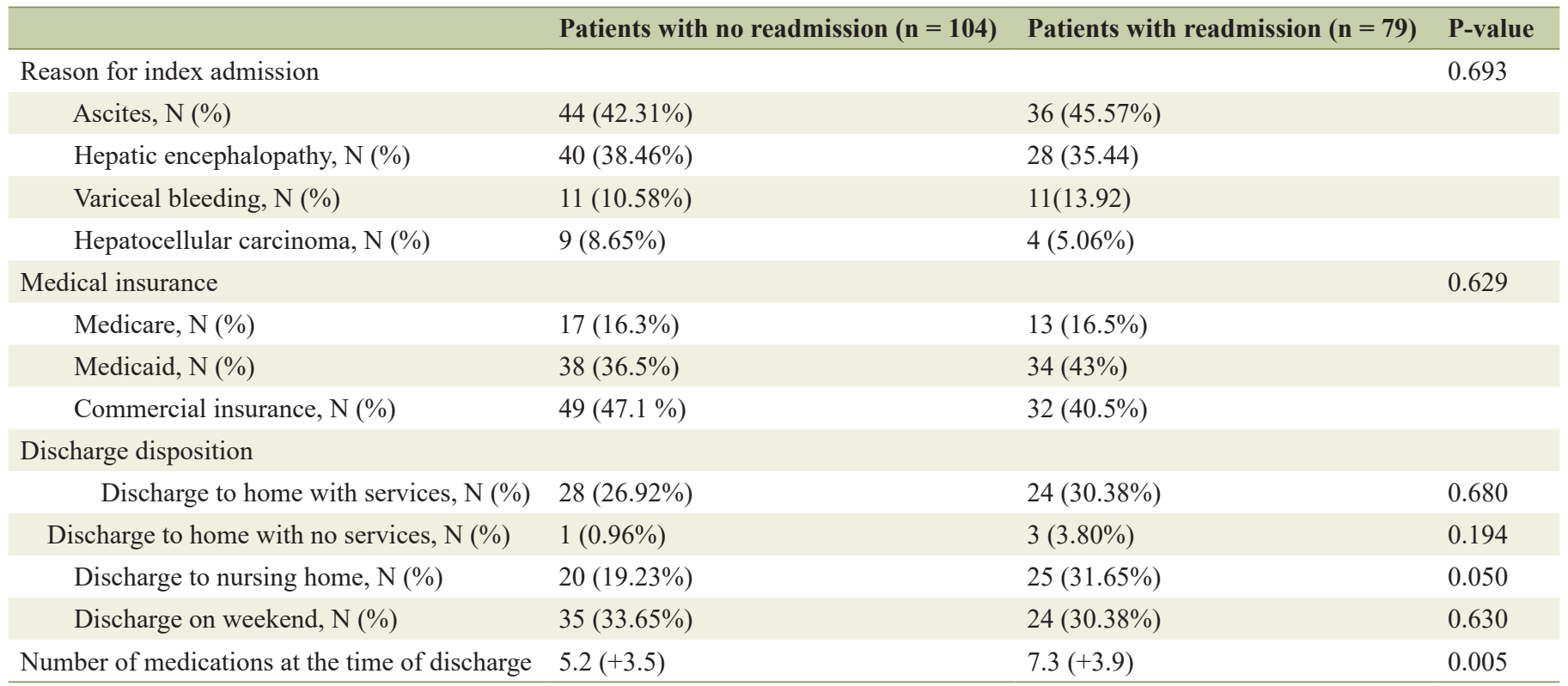

In the study group of patients with readmissions, we wanted to identify those at highest risk for readmission. Hence, we identified patients with more than four hospital visits during the study period and analyzed them further. We noted that $31 \%$ $(n=31 / 79)$ of patients contributed towards $61 \%(176 / 212)$ of hospital visits (Table 6). Implementation of measures in this high-risk group may subsequently reduce the readmission rates.
We performed a multivariate analysis for predictors of readmission in patients with liver cirrhosis (Table 7). Alcohol use within 1 month of admission, number of medications prescribed at the time of discharge and platelet count were found to be independent predictors for liver cirrhosis-related readmission. Alcohol use can lead to thrombocytopenia; however, lower platelet count predicted readmission independent of patient's alcohol use. Discharge to nursing home was not

Table 4. Sub-Analysis of Reason for Hospitalization for Patients in Readmission Group

\begin{tabular}{lll}
\hline Reason for hospitalization & $\begin{array}{l}\text { Index hospitalization for } \\
\text { patients with readmission }\end{array}$ & $\begin{array}{l}\text { Subsequent hospitalization for } \\
\text { patients with readmission }\end{array}$ \\
\hline Ascites, N (\%) & $36(45.5 \%)$ & $94(44.97 \%)$ \\
Hepatocellular carcinoma, N (\%) & $4(5.06 \%)$ & $9(4.3 \%)$ \\
Hepatic encephalopathy, N (\%) & $28(35.44 \%)$ & $90(43.06 \%)$ \\
Variceal bleeding, N (\%) & $11(13.92 \%)$ & $16(7.65 \%)$ \\
\hline
\end{tabular}


Table 5. Post-Hospital Discharge Follow-Up and the Post-Discharge 90-Day Mortality

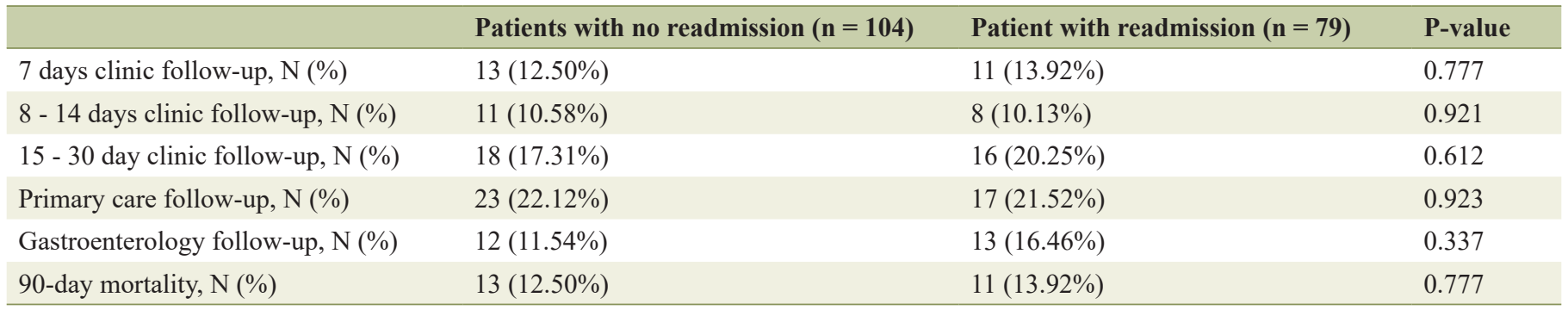

Table 6. Number of Unique Patients Contributing Towards Cumulative Hospital Visits

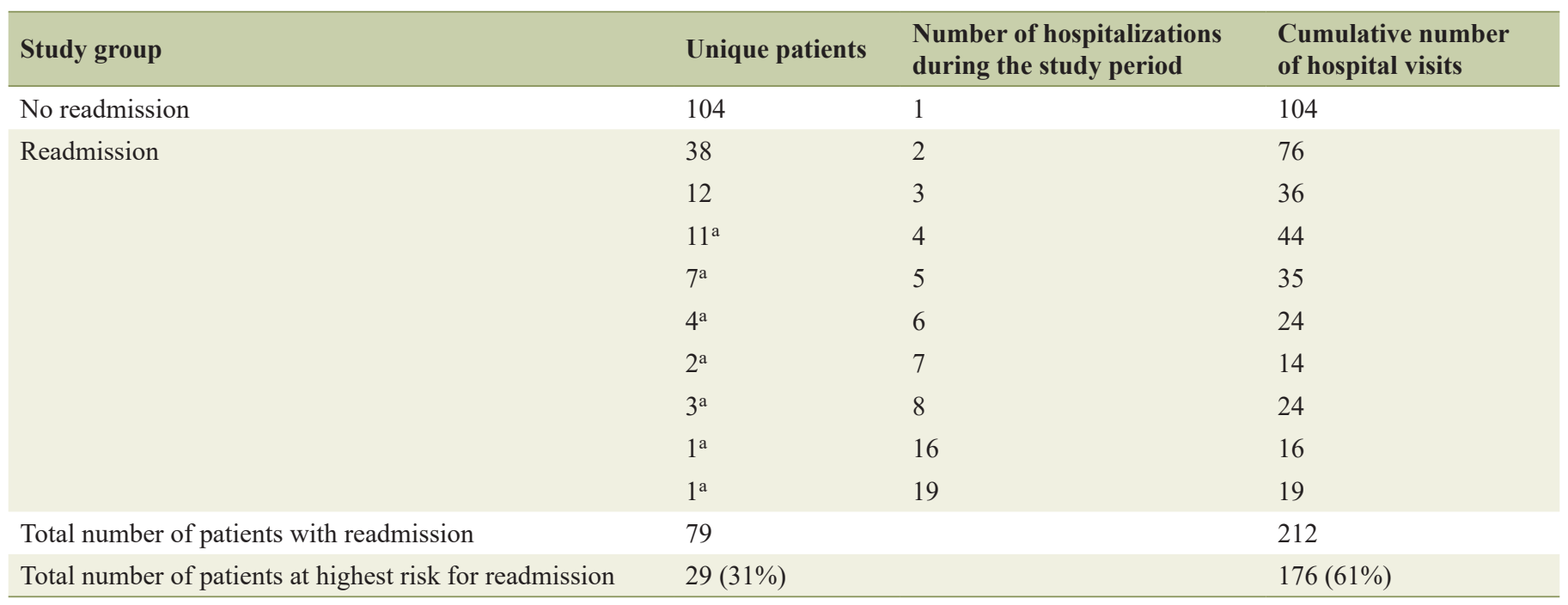

apatients at the highest risk for readmission who had four or more hospital visits during the study period.

an independent risk factor for readmission on multi-variate analysis.

\section{Discussion}

Reducing hospital readmission rates has attracted the policymakers as a way of reducing health care expenditure and improving the quality of health care delivered. Under the Affordable Care Act, CMS in 2012 decided to impose financial penalties on hospitals with higher readmission rates. Liver cirrhosis-related readmissions are currently not included in the HRRP program; however, given the positive results with current medical conditions subjected to HRRP as well as greater

Table 7. Predictors of 30-Day Readmission in Patients With Cirrhosis Identified by Multivariate Analysis

\begin{tabular}{ll} 
Variables & P-value \\
\hline Alcohol use within 1 month of admission & 0.016 \\
Discharge to a nursing home & 0.531 \\
Number of medications at the time of discharge & 0.039 \\
Platelet count & 0.049 \\
\hline
\end{tabular}

scrutiny of readmissions by policymakers, it can certainly be considered for the future programs. Though it is debatable if readmissions in patients with cirrhosis are preventable, studies have tried to identify risk factors which may be modifiable and help prevent unplanned readmissions. Unplanned readmission is a more complex problem than just a marker of poor health care delivery. Several factors other than disease process itself, like lack of social support, socio-economic status, lack of health insurance and level of education play a role in increasing the risk of readmission. Nevertheless, readmissions in patients with cirrhosis have a negative impact on patient's overall health, quality of life as well as equally the family. Hence, it is important to find ways to reduce readmissions in patients with cirrhosis.

In our study, 30-day readmission rate among our patients with cirrhosis was $26.5 \%$. Risk factors for readmissions included higher CPT class, a greater number of medications at discharge, lower platelet count and active alcohol use. Readmission rates in patients with cirrhosis may get impacted due to other coexisting medical conditions like CHF and hence can create a bias while studying predictors of liver cirrhosis-related readmission. Thus, in our study we included patients with liver cirrhosis only as a reason for the initial hospitalization or readmission, excluding any other non-cirrhosis reason for re- 
admission. Additionally, to understand patient characteristics influencing readmissions, we included unique patients rather multiple hospital visits for the patient who had readmission.

In general, advanced age, regardless of other medical comorbidities, is a significant independent risk factor that contributes towards readmissions. The majority of admissions to hospitals are estimated to be in age groups of 65 years and above [15]. However, given the increased prevalence of liver cirrhosis at an earlier age, mean age of patients in our study was 58.6 years, which does corroborate with the findings in the other studies [16]. In our study, age was not a predictor of readmission related to liver cirrhosis and this finding is similar to other studies [17].

Patients with higher MELD score are at risk for the readmission [7]. In our study population, patients in readmission group did have a higher MELD score and MELD-Na score as compared to patients with no readmission. However, this difference did not reach statistical significance. We noticed no significant difference in serum chemistry parameters like bilirubin, creatinine and prothrombin time among the two groups and hence the statistically insignificant MELD and MELD-Na score between the two groups. However, higher CPT class was a risk factor for the readmission. Uncontrolled ascites, nutritional status and hepatic encephalopathy are factors included in CPT score that are not accounted for in the MELD scoring system. In our study, we found that patients with the CPT class $\mathrm{C}$ were at higher risk for readmission. Readmission group in our study had no patient with CPT class A, hence reinforcing the association between higher CPT class and risk of readmission.

Thrombocytopenia in patients with chronic liver disease results from portal hypertension-induced hypersplenism and depressed thrombopoietin level [18]. There may be no correlation between hepatic dysfunction and platelet count in patients with liver cirrhosis [19]. However, lower platelet count has been identified as a risk factor for the readmission in patients with liver cirrhosis [19]. We also found thrombocytopenia as an independent risk factor for liver cirrhosis-related readmission.

Early outpatient follow-up and prompt recognition of factors accounting for the hospitalization may reduce the readmission rates. Studies have shown beneficial effects of followup within 7 days of hospital discharge [20]. In our study, we did not find any difference in the readmission rates in patients with 7 days, 8 - 14 days or 15 - 30 days follow-up after hospitalization. Overall, we had $40 \%$ of patient follow-up within 1 month in the no readmission group and $44 \%$ in the readmission group. Within 7 days, we had 13\% patient follow-up in the no readmission group and $14 \%$ in the readmission group. It is not clear if higher follow-up rates within a week in our patients would make a difference. Similar to other studies, we found no added advantage of having a patient follow-up with a gastroenterologist as compared to an internist [17].

Resources to manage patients with decompensated cirrhosis in an ambulatory setting are limited. Patients with volume overload and massive ascites who are on titrating doses of diuretics need close monitoring. Similarly, patients with history of hepatic encephalopathy need close monitoring of factors that can precipitate encephalopathy. Patients who reside in a nurs- ing home are continuously under nursing care and hence have a better chance of recognizing a decompensation or other complications early in patients with cirrhosis. This early recognition of problems may contribute towards higher trend of readmissions in patients at nursing homes as noted in our study.

Patients in our study who were discharged with more than seven medications were more likely to have readmission related to liver cirrhosis. Pharmacological interactions and risk of non-compliance can explain increasing risk of readmission with more medications at the time of discharge. Our results are similar to findings also reported by Volk et al [7]. Patients taking a greater number of medications may mean having more co-morbid conditions or having uncontrolled comorbid conditions requiring more medications. As seen in Table 1, except for hepatitis B which had an overall low number of patients, there was no significant difference in the number of comorbid conditions in the two groups. Patients who take a greater number of medications require close attention and monitoring, which may not be possible in patients with cirrhosis especially who have hepatic encephalopathy. Similar to other studies [8], hepatic encephalopathy and ascites were the two most common cirrhosis-related complications leading to the admission in our patients (Table 4). This may suggest a greater number of patients who required diuresis for ascites management developed electrolyte imbalance leading to hepatic encephalopathy. However, there was no difference in serum sodium and potassium levels between the two groups.

Chronic alcohol use is a significant risk factor for developing cirrhosis. In United States, alcohol accounts for approximately $36 \%$ of cases with cirrhosis. Hispanic patients constitute the highest proportion of alcohol-related cirrhosis population. Given the increasing trends of alcohol misuse among young people, prevalence of alcoholic cirrhosis is on the rise [2]. Hence, alcohol misuse significantly contributes towards the overall burden of chronic liver disease. In our study as well, we found a significantly higher number of patients with active alcohol use in the readmission group as compared to no readmission group ( $55 \%$ vs. $33 \%, \mathrm{P}=0.002)$.

Limitations of our study include retrospective design and single center data. We included patient's admission to our facility only and did not take admissions to other facility into account. Other important risk factors for readmission like social support, level of education, socio-economic status could not be looked at due to retrospective design of the study, and further studies are needed to explore the impact of these important social factors.

In conclusion, this study depicts that readmission in patients with cirrhosis are common. Platelet count at discharge, history of alcohol use and more than seven medications at discharge in Hispanic and African American patients admitted for cirrhosis are independent predictors of readmission. Only some of these risk factors are possibly modifiable. Unplanned readmission is a more complex problem than just a marker of poor health care delivery. Social factors like lack of family support, socio-economic status, lack of health insurance and level of education, all play an important role in the overall well-being of the patients with cirrhosis. Studies with larger number of patients and studying broader aspects of readmission are needed to guide future interventions to reduce readmission rate and 
health care costs related to cirrhosis readmissions.

\section{Acknowledgments}

None to declare.

\section{Financial Disclosure}

None to declare.

\section{Conflict of Interest}

The authors have no conflict of interest to disclose.

\section{Informed Consent}

Informed consent was waived by the Institutional Review Boards of BronxCare Hospital System due to the retrospective nature of this study.

\section{Author Contributions}

MI, CC, HH and MA collected the data. HP and VV drafted the manuscript. BB critically reviewed the manuscript. JM contributed to the study design and critically reviewed the manuscript.

\section{References}

1. United States Census Bureau. Hispanic Heritage Month 2018. In; 2018, September 13.

2. Moon AM, Singal AG, Tapper EB. Contemporary epidemiology of chronic liver disease and cirrhosis. Clin Gastroenterol Hepatol. 2019.

3. Heron M. Deaths: Leading Causes for 2016. Natl Vital Stat Rep. 2018;67(6):1-77.

4. Scaglione S, Kliethermes S, Cao G, Shoham D, Durazo R, Luke A, Volk ML. The epidemiology of cirrhosis in the United States: a population-based study. J Clin Gastroenterol. 2015;49(8):690-696.

5. Tapper EB, Finkelstein D, Mittleman MA, Piatkowski G, Lai M. Standard assessments of frailty are validated predictors of mortality in hospitalized patients with cirrhosis.
Hepatology. 2015;62(2):584-590.

6. Kim WR, Brown RS, Jr., Terrault NA, El-Serag H. Burden of liver disease in the United States: summary of a workshop. Hepatology. 2002;36(1):227-242.

7. Volk ML, Tocco RS, Bazick J, Rakoski MO, Lok AS. Hospital readmissions among patients with decompensated cirrhosis. Am J Gastroenterol. 2012;107(2):247-252.

8. Shaheen AA, Nguyen HH, Congly SE, Kaplan GG, Swain MG. Nationwide estimates and risk factors of hospital readmission in patients with cirrhosis in the United States. Liver Int. 2019;39(5):878-884.

9. Jencks SF, Brock JE. Hospital accountability and population health: lessons from measuring readmission rates. Ann Intern Med. 2013;159(9):629-630.

10. (CMS) CfMMS. Hospital Readmissions Reduction Program (HRRP). 2012.

11. Chamberlain RS, Sond J, Mahendraraj K, Lau CS, Siracuse BL. Determining 30-day readmission risk for heart failure patients: the readmission after heart failure scale. Int J Gen Med. 2018;11:127-141.

12. Ziaeian B, Fonarow GC. The Prevention of Hospital Readmissions in Heart Failure. Prog Cardiovasc Dis. 2016;58(4):379-385.

13. Tapper EB, Halbert B, Mellinger J. Rates of and Reasons for Hospital Readmissions in Patients With Cirrhosis: A Multistate Population-based Cohort Study. Clin Gastroenterol Hepatol. 2016;14(8):1181-1188 e1182.

14. Orman ES, Ghabril M, Emmett TW, Chalasani N. Hospital readmissions in patients with cirrhosis: a systematic review. J Hosp Med. 2018;13(7):490-495.

15. Dixon T, Shaw M, Frankel S, Ebrahim S. Hospital admissions, age, and death: retrospective cohort study. BMJ. 2004;328(7451): 1288 .

16. Majc D, Tepes B. The impact of outpatient clinical care on the survival and hospitalization rate in patients with alcoholic liver cirrhosis. Radiol Oncol. 2018;52(1):75-82.

17. Seraj SM, Campbell EJ, Argyropoulos SK, Wegermann K, Chung RT, Richter JM. Hospital readmissions in decompensated cirrhotics: Factors pointing toward a prevention strategy. World J Gastroenterol. 2017;23(37):6868-6876.

18. Mitchell O, Feldman DM, Diakow M, Sigal SH. The pathophysiology of thrombocytopenia in chronic liver disease. Hepat Med. 2016;8:39-50.

19. Djordjevic J, Svorcan P, Vrinic D, Dapcevic B. [Splenomegaly and thrombocytopenia in patients with liver cirrhosis]. Vojnosanit Pregl. 2010;67(2):166-169.

20. Jackson C, Shahsahebi M, Wedlake T, DuBard CA. Timeliness of outpatient follow-up: an evidence-based approach for planning after hospital discharge. Ann Fam Med. 2015;13(2):115-122. 